\title{
About the Kerr Nature of the Stellar-mass Black Hole in GRS 1915+105
}

\author{
Yuexin Zhang ${ }^{1}$, Askar B. Abdikamalov ${ }^{1}$, Dimitry Ayzenberg ${ }^{1}$, Cosimo Bambi $^{1}$ (1) Thomas Dauser $^{2}$, Javier A. García ${ }^{2,3}$, and \\ Sourabh Nampalliwar ${ }^{4}$ \\ ${ }^{1}$ Center for Field Theory and Particle Physics and Department of Physics, Fudan University, 200438 Shanghai, People's Republic of China; bambi@fudan.edu.cn \\ ${ }^{2}$ Remeis Observatory \& ECAP, Universität Erlangen-Nürnberg, D-96049 Bamberg, Germany \\ ${ }^{3}$ Cahill Center for Astronomy and Astrophysics, California Institute of Technology, Pasadena, CA 91125, USA \\ ${ }^{4}$ Theoretical Astrophysics, Eberhard-Karls Universität Tübingen, D-72076 Tübingen, Germany \\ Received 2019 January 23; revised 2019 March 4; accepted 2019 March 8; published 2019 April 12
}

\begin{abstract}
We employ the accretion disk reflection model RELXILL_NK to test the spacetime geometry around the stellar-mass black hole in GRS 1915+105. We adopt the Johannsen metric with the deformation parameters $\alpha_{13}$ and $\alpha_{22}$, for which the Kerr solution is recovered when $\alpha_{13}=\alpha_{22}=0$. We analyze a NuSTAR observation of 2012, obtaining vanishing and non-vanishing values of the deformation parameters depending on the astrophysical model adopted. Similar difficulties were not found in our previous tests with other sources. The results of this work can shed light on the choice of sources suitable for testing the Kerr metric using X-ray reflection spectroscopy and on the parts of our reflection models that more urgently require improvement.
\end{abstract}

Key words: accretion, accretion disks - black hole physics - gravitation

\section{Introduction}

Astrophysical black holes are commonly thought to be the Kerr black holes predicted by Einstein's theory of general relativity (Narayan 2005; Bambi 2018). Nevertheless, it is important to bear in mind that Einstein's gravity has been extensively tested only in weak gravitational fields (Will 2014). There are many alternative and modified theories of gravity that have the same predictions as Einstein's gravity in the weak field regime but show deviations from general relativity when gravity becomes strong. Astrophysical black holes are an ideal laboratory for testing Einstein's theory of general relativity in the strong-field regime.

Nowadays, there are two lines of research to test black holes and strong gravity: (i) the study of the properties of electromagnetic radiation emitted by material orbiting close to a black hole (Bambi et al. 2016; Cardoso \& Gualtieri 2016; Bambi 2017; Krawczynski 2018), and (ii) the study of the gravitational wave signal emitted by black hole systems (Yunes \& Siemens 2013; Abbott et al. 2016; Yagi \& Stein 2016; Yunes et al. 2016). The two approaches are complementary because they probe different sectors of the theory. Electromagnetic techniques can, strictly speaking, only test the motion of massless and massive particles in the strong gravity region. The gravitational wave spectrum is sensitive to the evolution of the spacetime metric and thus can test the strong-field, highly dynamical regime. In the present work, we consider one of the electromagnetic techniques for black hole tests: X-ray reflection spectroscopy.

$\mathrm{X}$-ray reflection spectroscopy is potentially a powerful technique to test the Kerr nature of astrophysical black holes with electromagnetic radiation (Schee \& Stuchlík 2009; Bambi 2013a, 2013b; Johannsen \& Psaltis 2013; Jiang et al. 2015; Ni et al. 2016; Zhou et al. 2016; Bambi et al. 2018). This technique is based on the study of the reflection spectrum of accretion disks (Fabian et al. 2000; Reynolds 2014). The accretion disk of black holes emits thermal photons that can have inverse Compton scattering off free electrons in the socalled "corona", which is a hot, usually compact and optically thin, medium close to the compact object. A fraction of the
Comptonized photons illuminate the disk, producing a reflection spectrum with some emission lines. The most prominent features in the reflection spectrum are usually the iron $\mathrm{K} \alpha$ line around $6 \mathrm{keV}$ and the Compton hump at $10-30 \mathrm{keV}$. The observed reflection spectrum, and the iron $\mathrm{K} \alpha$ line in particular, are strongly affected by relativistic effects occurring in the strong gravity region around the black hole. In the presence of the correct astrophysical model and highquality data, we can study the features of the reflection spectrum and test the nature of the compact object.

Recently, we have developed the reflection model RELXILL_NK to probe the spacetime metric around astrophysical black holes and test the Kerr black hole hypothesis using $\mathrm{X}$-ray reflection spectroscopy (Bambi et al. 2017; Abdikamalov et al. 2019). RELXILL_NK is the natural extension of the RELXILL model (Dauser et al. 2013; García et al. 2014) to nonKerr spacetimes. In RELXILL_NK, the spacetime is described by a parametric black hole metric in which a set of "deformation parameters" is introduced to quantify possible deviations from the Kerr solution. By comparing X-ray data of astrophysical black holes with the theoretical predictions of RELXILL_NK, we can measure the values of these deformation parameters and check whether they vanish, as is required by Einstein's theory.

In the past year, we have analyzed a few sources with RELXILL_NK. In the case of supermassive black holes, we have tested 1H0707-495 with XMM-Newton and NuSTAR data (Cao et al. 2018), Ark 564 and Mrk 335 with Suzaku data (Choudhury et al. 2018; Tripathi et al. 2018a), and MCG-6-30-15 with combined data of XMM-Newton and NuSTAR (Tripathi et al. 2018b). In Tripathi et al. (2019), we have presented the analysis of Suzaku data of seven "bare" active galactic nuclei (Ton S180， RBS 1124， Ark 120， Swift J0501.9-3239, 1H0419-577, PKS 0558-504, and Fairall 9), i.e., sources with no complicating intrinsic absorption. In the case of stellar-mass black holes, we have tested GX 339-4 with RXTE data (Wang$\mathrm{Ji}$ et al. 2018) and GS 1354-645 with NuSTAR data (Xu et al. 2018). In all these studies, we have found that the measurements of the value of the deformation parameters are consistent with zero at $1 \sigma$ or $2 \sigma$; that is, our results are consistent with the 
hypothesis that the spacetime metric around all these objects is described by the Kerr solution within the statistical uncertainties of our measurements; systematic uncertainties are more difficult to estimate and work is underway. The constraints obtained from MCG-6-30-15, GS 1354-645, and some bare active galactic nuclei appear quite stringent, and we have shown how imposing unjustified ad hoc emissivity profiles would completely spoil our results, suggesting that our current theoretical model is good enough to test Einstein's gravity with these sources ( $\mathrm{Xu}$ et al. 2018).

Here we continue our program of testing the Kerr black hole hypothesis with RELXILL_NK and we present the study of a new source, GRS $1915+105$, which is a binary system of a stellar-mass black hole with a low-mass companion star. We analyze a NuSTAR observation of 2012, hoping to get strong constraints on the deformation parameters, analogous to what was obtained for GS 1354-645. Like the latter, GRS 1915 +105 has properties that are supposed to help obtain good constraints on its strong gravity region: its spin parameter is high, so the inner edge of the disk can be very close to the black hole, and the viewing angle is relatively high as well, thus maximizing the relativistic effects of Doppler boosting and light bending. Since it is a stellar-mass black hole, the source is bright and we have a good statistics. NuSTAR data are also suitable for this kind of test, as we can measure the spectrum up to $80 \mathrm{keV}$ and there is no pile-up problem. However, we meet a problem in recovering the Kerr metric. More specifically, we find non-vanishing deformation parameters when we employ the model adopted in Miller et al. (2013), where the authors study this NuSTAR observation assuming the Kerr metric. We try to change the intensity profile, but we consistently do not recover the Kerr solution. When we add a non-relativistic reflection component, we recover Kerr when the intensity profile is modeled with a power law, and we do not recover Kerr with a broken power law. In our previous tests, we had never met similar difficulties. We compare the results of this work with those of previous tests of the Kerr metric, and we discuss the differences between GRS 1915+105 and the other sources.

The content of the paper is as follows. In Section 2, we review our method to test the Kerr black hole hypothesis with the reflection model RELXILL_NK. In Section 3, we present the observation and how we reduced the data. Section 4 is devoted to the data analysis and we show the best-fit values and the constraints on the deformation parameters. In Section 5, we discuss our results and we compare them with those obtained in other studies. Throughout the paper, we employ a metric with signature $(-+++)$ and units in which $G_{\mathrm{N}}=c=1$.

\section{Testing the Kerr Hypothesis with RELXILL NK}

The reflection spectrum of accretion disks around black holes originates from the illumination of the accretion disk by Comptonized photons from the corona. From the comparison of the theoretical predictions with observational data, it is possible to infer the properties of the system. Our disk's reflection model for non-Kerr spacetimes is called RELXILL_NK and was presented in Bambi et al. (2017) and Abdikamalov et al. (2019). It is the natural extension of the RELXILL model (Dauser et al. 2013; García et al. 2014), in which the background metric is assumed to be described by the Kerr solution. RELXILL itself is the result of the merger of two models: XILLVER and RELCONV. XILLVER is a purely atomic model to calculate the reflection spectrum in the rest-frame of the gas of the accretion disk (García \& Kallman 2010; García et al. 2013). RELCONV is a convolution model and transforms the reflection spectrum calculated by XILLVER into that detected far from the source taking all relativistic effects (Doppler boosting, gravitational redshift, and light bending) into account (Dauser et al. 2010). In RELXILL_NK, we have extended the convolution model RELCONV in order to calculate the detected spectrum in the case of a non-Kerr spacetime.

In what follows, we assume that the geometry of the spacetime is described by the Johannsen metric (Johannsen 2013) ${ }^{5}$. In Boyer-Lindquist-like coordinates, the line element reads

$$
\begin{aligned}
d s^{2}= & -\frac{\tilde{\Sigma}\left(\Delta-a^{2} A_{2}^{2} \sin ^{2} \theta\right)}{B^{2}} d t^{2}+\frac{\tilde{\Sigma}}{\Delta} d r^{2}+\tilde{\Sigma} d \theta^{2} \\
& -\frac{2 a\left[\left(r^{2}+a^{2}\right) A_{1} A_{2}-\Delta\right] \tilde{\Sigma} \sin ^{2} \theta}{B^{2}} d t d \phi \\
& +\frac{\left[\left(r^{2}+a^{2}\right)^{2} A_{1}^{2}-a^{2} \Delta \sin ^{2} \theta\right] \tilde{\Sigma} \sin ^{2} \theta}{B^{2}} d \phi^{2}
\end{aligned}
$$

where $M$ is the black hole mass, $a=J / M, J$ is the black hole spin angular momentum, $\tilde{\Sigma}=\Sigma=f$, and

$$
\begin{aligned}
& \Sigma=r^{2}+a^{2} \cos ^{2} \theta, \quad \Delta=r^{2}-2 M r+a^{2}, \\
& B=\left(r^{2}+a^{2}\right) A_{1}-a^{2} A_{2} \sin ^{2} \theta .
\end{aligned}
$$

The functions $f, A_{1}, A_{2}$, and $A_{5}$ are defined as

$$
\begin{aligned}
f & =\sum_{n=3}^{\infty} \epsilon_{n} \frac{M^{n}}{r^{n-2}}, \quad A_{1}=1+\sum_{n=3}^{\infty} \alpha_{1 n}\left(\frac{M}{r}\right)^{n}, \\
A_{2} & =1+\sum_{n=2}^{\infty} \alpha_{2 n}\left(\frac{M}{r}\right)^{n}, \quad A_{5}=1+\sum_{n=2}^{\infty} \alpha_{5 n}\left(\frac{M}{r}\right)^{n} .
\end{aligned}
$$

$\left\{\epsilon_{n}\right\},\left\{\alpha_{1 n}\right\},\left\{\alpha_{2 n}\right\}$, and $\left\{\alpha_{5 n}\right\}$ are four infinite sets of deformation parameters without constraints from the Newtonian limit and weak field experiments, and the Kerr metric is recovered when all deformation parameters vanish. In this paper, we will only focus on the deformation parameters $\alpha_{13}$ and $\alpha_{22}$, as they are the two with the strongest impact on the reflection spectrum (Bambi et al. 2017). In what follows, we will consider the possibility that one of the two deformation parameters may be non-vanishing and we will try to infer its value from the data of GRS $1915+105$. First, we will try to measure $\alpha_{13}$ assuming that $\alpha_{22}=0$ and then we will consider the opposite case, namely $\alpha_{13}=0$ and we try to measure the value of $\alpha_{22}$. The possibility of two variable deformation parameters at the same time is beyond the capabilities of our current version of RELXILL_NK.

Note that, in order to avoid spacetimes with pathological properties, we have to impose some restrictions on the values of the spin parameter $a_{*}=a / M$ and of the deformation parameters $\alpha_{13}$ and $\alpha_{22}$. As in the case of the Kerr spacetime, we require that $\left|a_{*}\right| \leqslant 1$, because for $\left|a_{*}\right|>1$, there is no black hole but a naked singularity. As discussed in Johannsen (2013)

\footnotetext{
5 The Johannsen metric is not a black hole solution of any modified theory of gravity but simply a parametric black hole metric aiming at describing a spacetime with possible deviations from the Kerr solution. Here we employ the Johannsen metric with the spirit to perform a null experiment and check whether astrophysical observations require that the value of the deformation parameters is consistent with zero as requested by the Kerr hypothesis.
} 

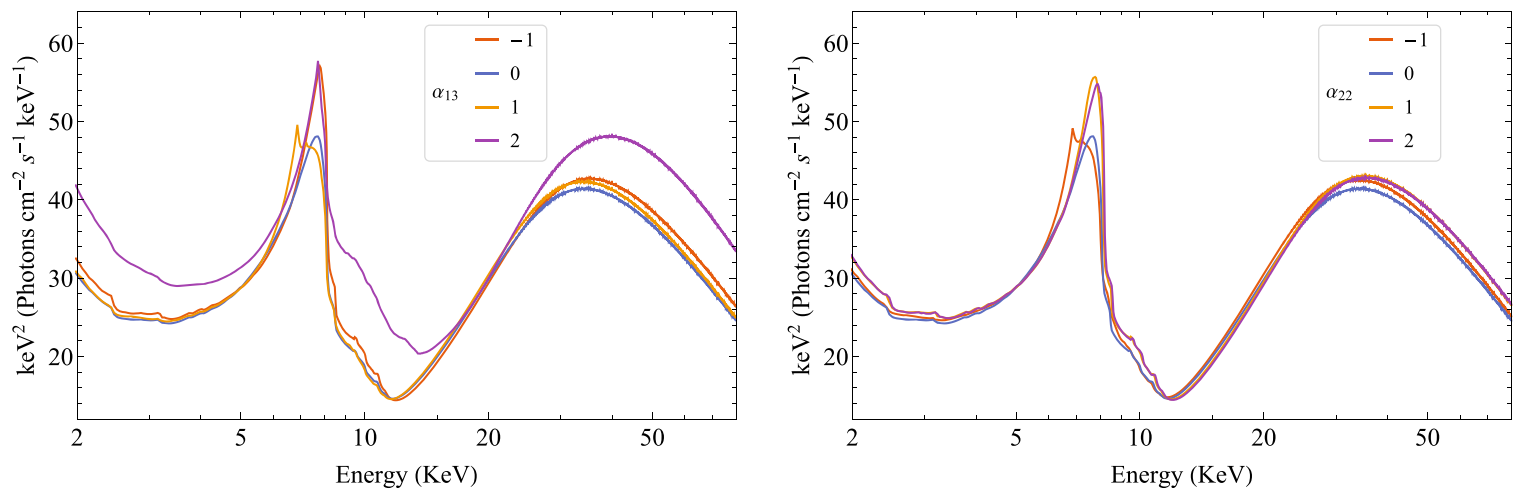

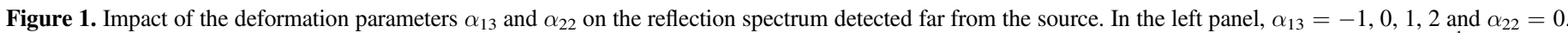

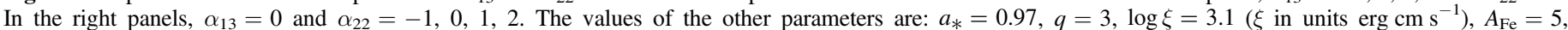
and $i=60^{\circ}$.

and Tripathi et al. (2018a), we also have to impose the following restrictions on $\alpha_{13}$ and $\alpha_{22}$ :

$$
\begin{gathered}
\alpha_{13}>-\frac{1}{2}\left(1+\sqrt{1-a_{*}^{2}}\right)^{4}, \\
-\left(1+\sqrt{1-a_{*}^{2}}\right)^{2}<\alpha_{22}<\frac{\left(1+\sqrt{1-a_{*}^{2}}\right)^{4}}{a_{*}^{2}} .
\end{gathered}
$$

Additional details on the astrophysical model employed in RELXILL_NK can be found in Bambi et al. (2017) and Abdikamalov et al. (2019). Here, we remind the reader that the accretion disk is assumed to be infinitesimally thin and on the equatorial plane; i.e., orthogonal to the black hole spin. The gas of the accretion disk follows nearly geodesic, equatorial, circular orbits. For thin accretion disks, the inner edge can be at or outside the innermost stable circular orbit (or ISCO); in the present work, we make the standard assumption to fix it at the ISCO radius. The emissivity profile of the accretion disk can be modeled with a power law (i.e., the intensity is proportional to $1 / r^{q}$, where $q$ is some emissivity index) or a broken power law (i.e., the intensity is proportional to $1 / r^{q}$ for $r<R_{\mathrm{br}}$ and to $1 / r^{q_{\text {out }}}$ for $r>R_{\mathrm{br}}$, where $q_{\text {in }}$ and $q_{\text {out }}$ are the inner and the outer emissivity index, respectively, and $R_{\mathrm{br}}$ is called the breaking radius). The ionization of the accretion disk is described by a single ionization parameter $\xi$ and the composition of the accretion disk is taken into account by the iron abundance $A_{\mathrm{Fe}}$. An important parameter in the model is the viewing angle $i$, namely the angle between our line of sight and the spin of the black hole. Figure 1 shows the typical reflection spectrum as calculated by RELXILL_NK for a few values of $\alpha_{13}$ and $\alpha_{22}$, respectively. Note that the reflection spectrum of the disk does not directly depend on the black hole mass (the mass, however, regulates the temperature of the disk and, in turn, the ionization parameter $\xi$ ). Therefore, unlike the continuum-fitting method, $\mathrm{X}$-ray reflection spectroscopy does not need any independent measurement of the black hole mass to fit the spectrum of the source.

\section{Observations and Data Reduction}

GRS $1915+105$ is quite a special source. While it is a lowmass X-ray binary (i.e., the mass of the companion star is less than a few solar masses), it has been a persistent X-ray source since 1992. This is probably due to its large accretion disk, which is capable of providing a sufficiently high mass transfer at any time.
NuSTAR observed GRS 1915+105 on 2012 July 3 for approximately $60 \mathrm{ks}$. This observation was analyzed for the first time in Miller et al. (2013), where the authors-assuming the Kerr metric-measured a spin parameter $a_{*}=0.98 \pm 0.01$ at $1 \sigma$ statistical error.

In our analysis, we employ Xspec v12.10.0 (Arnaud 1996). We process the data from both the FPMA and FPMB instruments using nupipeline v0.4.3 with the standard filtering criteria and the NuSTAR CALDB version 20180419. We use the nuproducts routine to extract source spectra, responses, and background spectra. Source spectra are extracted from a circular region of radius $90^{\prime \prime}$. Background spectra are extracted from regions of equivalent size on each detector. All spectra are grouped to a minimum of 30 counts before analysis to ensure the validity of the $\chi^{2}$ fit statistics. After all efficiencies and screening, the net exposure time for the resultant spectra is $14.85 \mathrm{ks}$ for FPMA, and $15.31 \mathrm{ks}$ for FPMB.

Assuming the black hole mass $M_{\mathrm{BH}}=(10.1 \pm 0.6) M_{\odot}$ and distance $D=11 \mathrm{kpc}$ (Steeghs et al. 2013), the accretion luminosity of the black hole is $0.23 \pm 0.04$ in Eddington units. It is thus in the range in which the accretion disk is thought to be well described by the Novikov-Thorne model with the inner edge at the ISCO radius (Steiner et al. 2010; McClintock et al. 2014), which is the model employed in our analysis.

GRS $1915+105$ is a highly variable source. However, as shown in Figure 2, the source was quite stable during the 2012 NUSTAR observation, and therefore we do not need to take its variability into account in our spectral analysis.

\section{Spectral Analysis}

We start fitting the data with a power-law component with an exponential cut-off describing the corona spectrum (model 0 ). The Xspec model is TBABS $*$ CUTOFFPL, where TBABS describes the Galactic absorption (Wilms et al. 2000) and CUTOFFPL is for the power-law component. The fit is poor, and we clearly see a broad iron line around $6 \mathrm{keV}$ and a Compton hump around $20 \mathrm{keV}$ (see the top panels in Figure 3).

We improve our model by adding a relativistic reflection component with RELXILL_NK. Throughout this paper, we employ the version 1.3.2 described in Abdikamalov et al. 

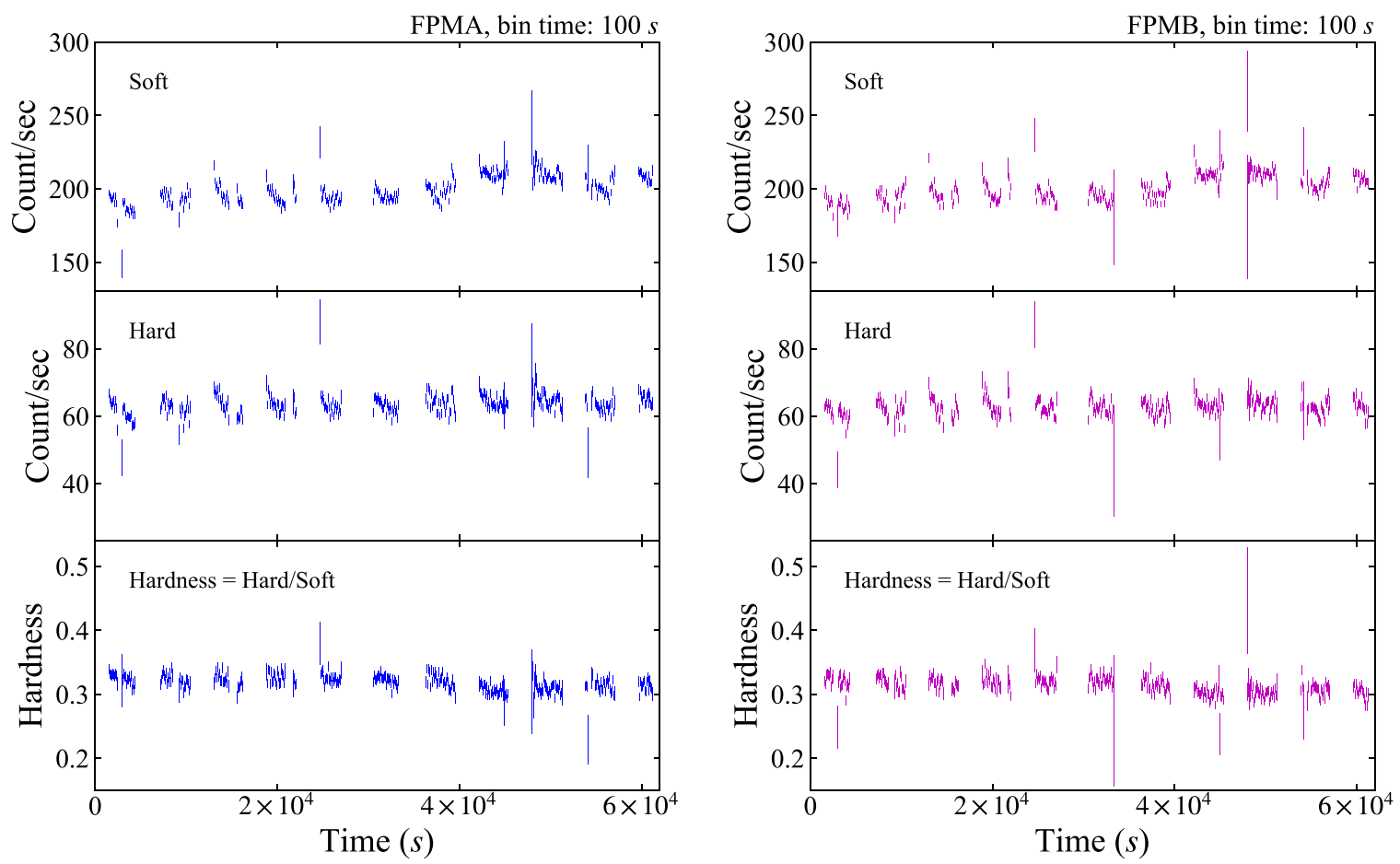

Figure 2. Light curves in the soft (3-10 keV) and hard (10-80 keV) bands of GRS 1915+105 on 2012 July 3 from FPMA (left panel) and FPMB (right panel) and temporal evolution of the hardness of the spectrum.
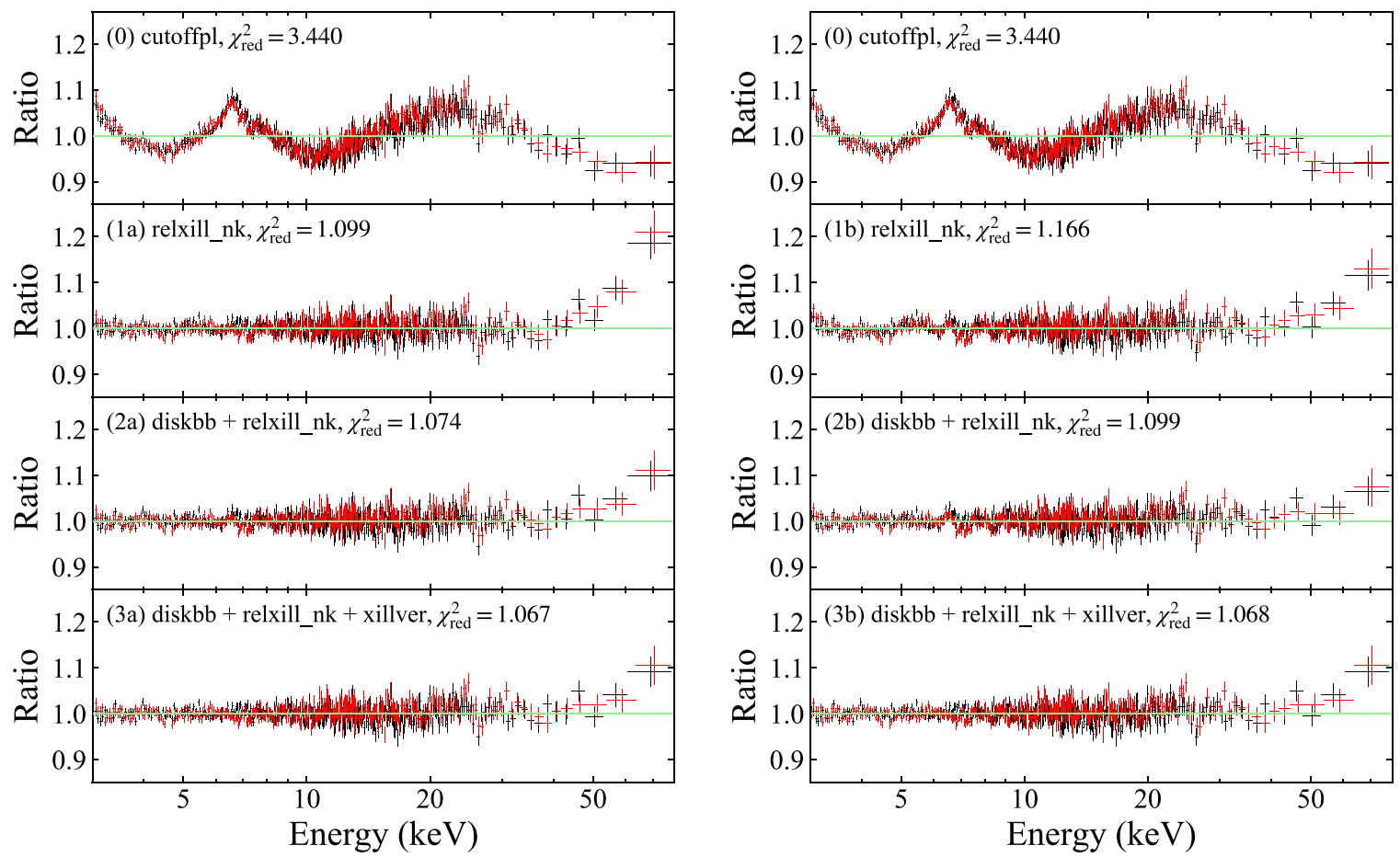

Figure 3. Data to best-fit model ratios for the fits with $\alpha_{13}$ free and $\alpha_{22}=0$ (left panels) and for those with $\alpha_{13}=0$ and $\alpha_{22}$ free (right panels) of the NuSTAR observation of 2012.

(2019) and which is available online. ${ }^{6}$ We have three models: model 1 in which $\alpha_{13}=\alpha_{22}=0$ (Kerr spacetime), model $1 a$ in which $\alpha_{13}$ free and $\alpha_{22}=0$, and model $1 b$ with $\alpha_{13}=0$ and $\alpha_{22}$ free. Figure 3 shows the ratio plots of models $1 a$ and $1 b$, where we can clearly see that the fits are significantly better

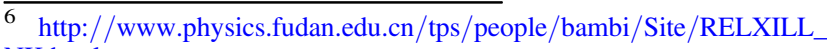
NK.html.
}

than model 0 . We still have an excess of counts at low (around $3 \mathrm{keV}$ ) and high (above $40 \mathrm{keV}$ ) energies.

We add a thermal component for the accretion disk, which is often present in the spectrum of GRS 1915+105. Again, we have three variants: model 2 in which we assume the Kerr metric, model $2 a$ in which $\alpha_{13}$ is free and $\alpha_{22}=0$, and model $2 b$ with $\alpha_{13}=0$ and $\alpha_{22}$ is free. For the disk's thermal spectrum, we use the Xspec model DISKBB (Mitsuda et al. 
Table 1

Summary of the Best-fit Values for Models 2, 3, and 3' (Kerr Spacetime with $\alpha_{13}=\alpha_{22}=0$ )

\begin{tabular}{|c|c|c|c|}
\hline $\begin{array}{l}\text { Model } \\
\text { TBABS }\end{array}$ & 2 & 3 & $3^{\prime}$ \\
\hline$N_{\mathrm{H}} / 10^{22} \mathrm{~cm}^{-2}$ & $8.93_{-0.06}^{+0.31}$ & $7.1_{-0.6}^{+0.6}$ & $8.1_{-0.4}^{+0.3}$ \\
\hline $\begin{array}{l}\text { DISKBB } \\
T_{\text {in }}[\mathrm{keV}]\end{array}$ & $0.4205_{-0.0014}^{+0.0011}$ & $0.427_{-0.025}^{+0.029}$ & $0.400_{-0.011}^{+0.016}$ \\
\hline \multicolumn{4}{|l|}{ RELXILL_NK } \\
\hline$q_{\text {in }}$ & $>9.8$ & $4.7_{-1.2}^{+4.3}$ & $<3.3$ \\
\hline$q_{\text {out }}$ & $=q_{\text {in }}$ & $=q_{\text {in }}$ & $>9.4$ \\
\hline$R_{\mathrm{br}}[M]$ & $\cdots$ & $\cdots$ & $1.71_{-0.07}^{+0.06}$ \\
\hline$i[\mathrm{deg}]$ & $75.59_{-0.16}^{+0.23}$ & $64_{-3}^{+9}$ & $75.9_{-0.9}^{+0.7}$ \\
\hline$a_{*}$ & $0.9875_{-0.0056}^{+0.0006}$ & $0.967_{-0.025}^{+0.012}$ & $>0.989$ \\
\hline$\alpha_{13}$ & $0^{\mathrm{a}}$ & $0^{\mathrm{a}}$ & $0^{\mathrm{a}}$ \\
\hline$\alpha_{22}$ & $0^{\mathrm{a}}$ & $0^{\mathrm{a}}$ & $0^{\mathrm{a}}$ \\
\hline $\log \xi$ & $3.025_{-0.014}^{+0.028}$ & $3.47_{-0.16}^{+0.25}$ & $3.04_{-0.03}^{+0.04}$ \\
\hline$A_{\mathrm{Fe}}$ & $0.907_{-0.081}^{+0.021}$ & $1.1_{-0.3}^{+0.8}$ & $0.67_{-0.07}^{+0.07}$ \\
\hline$\Gamma$ & $2.080_{-0.004}^{+0.004}$ & $1.89_{-0.08}^{+0.05}$ & $2.13_{-0.05}^{+0.03}$ \\
\hline$E_{\text {cut }}[\mathrm{keV}]$ & $60.6_{-1.0}^{+0.5}$ & $47_{-5}^{+6}$ & $69_{-8}^{+5}$ \\
\hline$R_{\mathrm{f}}$ & $0.228_{-0.011}^{+0.006}$ & $0.17_{-0.03}^{+0.07}$ & $0.27_{-0.04}^{+0.03}$ \\
\hline \multicolumn{4}{|l|}{ XILLVER } \\
\hline $\log \xi$ & $\cdots$ & $2.80_{-0.09}^{+0.14}$ & $2.30_{-0.15}^{+0.09}$ \\
\hline \multirow[t]{2}{*}{$\chi^{2} / \nu$} & $2630.40 / 2388$ & $2546.33 / 2386$ & $2537.82 / 2384$ \\
\hline & $=1.10151$ & $=1.06719$ & $=1.06452$ \\
\hline
\end{tabular}

Notes. The reported uncertainties correspond to the $90 \%$ confidence level for one relevant parameter.

${ }^{\mathrm{a}}$ Indicates that the parameter is frozen.

Table 2

Summary of the Best-fit Values for Models $2 a, 2 b, 3 a, 3 b, 3 a^{\prime}$, and $3 b^{\prime}$

\begin{tabular}{|c|c|c|c|c|c|c|}
\hline Model & $2 a$ & $2 b$ & $3 a$ & $3 b$ & $3 a^{\prime}$ & $3 b^{\prime}$ \\
\hline $\begin{array}{l}\text { TBABS } \\
N_{\mathrm{H}} / 10^{22} \mathrm{~cm}^{-2}\end{array}$ & $7.4_{-0.4}^{+0.4}$ & $8.69_{-0.36}^{+0.20}$ & $7.0_{-0.9}^{+0.6}$ & $7.1_{-0.6}^{+0.6}$ & $8.66_{-0.12}^{+0.13}$ & $8.1_{-0.3}^{+0.5}$ \\
\hline $\begin{array}{l}\text { DISKBB } \\
T_{\text {in }}[\mathrm{keV}]\end{array}$ & $0.418_{-0.020}^{+0.018}$ & $0.425_{-0.016}^{+0.010}$ & $0.43_{-0.03}^{+0.03}$ & $0.427_{-0.022}^{+0.023}$ & $0.3537_{-0.0213}^{+0.0012}$ & $0.402_{-0.018}^{+0.014}$ \\
\hline \multicolumn{7}{|l|}{ RELXILL_NK } \\
\hline$q_{\text {in }}$ & $5.7_{-0.9}^{+1.4}$ & $>9.7$ & $3.3_{-0.9}^{+2.6}$ & $4.7_{-2.0}^{+3.0}$ & $0.2^{+1.2}$ & $4.2_{-2.1}^{+2.1}$ \\
\hline $\begin{array}{l}q_{\text {out }} \\
R_{\mathrm{b}}[M]\end{array}$ & $\begin{array}{c}=q_{\text {in }} \\
\ldots\end{array}$ & $\begin{array}{c}=q_{\text {in }} \\
\ldots\end{array}$ & $\begin{array}{c}=q_{\text {in }} \\
\ldots\end{array}$ & $\begin{array}{c}=q_{\text {in }} \\
\ldots\end{array}$ & $\begin{array}{c}7.3_{-1.5} \\
1.85^{+0.35}\end{array}$ & $\begin{array}{c}>8 \\
1.40^{+0.14}\end{array}$ \\
\hline$i[\mathrm{deg}]$ & $64.9_{-0.5}^{+0.4}$ & $74.5_{-0.4}^{+0.7}$ & $62.2_{-2.3}^{+1.2}$ & $64_{-3}^{+6}$ & $68.8_{-0.7}^{+0.06}$ & $77.8_{-5.5}^{+0.90}$ \\
\hline$a_{*}$ & $>0.993$ & $>0.995$ & $0.989_{0.010}$ & $0.971_{-0.071}^{+0.021}$ & $0.913_{-0.009}^{+0.014}$ & $>0.990$ \\
\hline$\alpha_{13}$ & $-0.50_{-0.01}^{+0.02}$ & $0 \star$ & $0.2_{-1.7}^{+0.1}$ & $0 \star$ & $-1.7_{-0.1}^{+0.4}$ & $0 \star$ \\
\hline$\alpha_{22}$ & $0 \star$ & $-0.13_{-0.01}^{+0.05}$ & $0 \star$ & $0.0_{-0.2}^{+0.7}$ & $0 \star$ & $0.21_{-0.12}^{+0.04}$ \\
\hline $\log \xi$ & $2.96_{-0.11}^{+0.05}$ & $3.04_{-0.07}^{+0.03}$ & $3.51_{-0.08}^{+0.14}$ & $3.47_{-0.17}^{+0.21}$ & $2.85_{-0.03}^{+0.07}$ & $3.05_{-0.04}^{+0.04}$ \\
\hline$A_{\mathrm{Fe}}$ & $1.4_{-0.4}^{+0.4}$ & $0.97_{-0.23}^{+0.18}$ & $1.01_{-0.16}^{+0.60}$ & $1.07_{-0.17}^{+1.03}$ & $0.560_{-0.017}^{+0.051}$ & $0.67_{-0.05}^{+0.12}$ \\
\hline$\Gamma$ & $2.01_{-0.03}^{+0.04}$ & $2.044_{-0.011}^{+0.015}$ & $1.889_{-0.030}^{+0.023}$ & $1.89_{-0.07}^{+0.05}$ & $2.261_{-0.034}^{+0.021}$ & $2.13_{-0.04}^{+0.05}$ \\
\hline$E_{\text {cut }}[\mathrm{keV}]$ & $51.7_{-2.9}^{+2.2}$ & $56.7_{-1.6}^{+6.5}$ & $47_{-3}^{+4}$ & $47_{-6}^{+5}$ & $87.2_{-1.8}^{+3.3}$ & $69_{-7}^{+7}$ \\
\hline$R_{\mathrm{f}}$ & $0.209_{-0.017}^{+0.015}$ & $0.202_{-0.013}^{+0.066}$ & $0.16_{-0.03}^{+0.07}$ & $0.17_{-0.03}^{+0.06}$ & $0.345_{-0.014}^{+0.024}$ & $0.273_{-0.021}^{+0.021}$ \\
\hline $\begin{array}{l}\text { XILLVER } \\
\log \xi\end{array}$ & $\cdots$ & $\cdots$ & $2.84_{-0.08}^{+0.15}$ & $2.80_{-0.09}^{+0.13}$ & $3.10_{-0.20}^{+0.08}$ & $2.29_{-0.16}^{+0.09}$ \\
\hline$\chi^{2} / \nu$ & $\begin{array}{c}2564.57 / 2387 \\
=1.07439\end{array}$ & $\begin{array}{c}2622.33 / 2387 \\
=1.09859\end{array}$ & $\begin{array}{c}2545.12 / 2385 \\
=1.06714\end{array}$ & $\begin{array}{c}2546.35 / 2385 \\
=1.06765\end{array}$ & $\begin{array}{c}2508.85 / 2383 \\
1.05281\end{array}$ & $\begin{array}{c}2535.54 / 2383 \\
1.06401\end{array}$ \\
\hline
\end{tabular}

Note. The reported uncertainties correspond to the $90 \%$ confidence level for one relevant parameter. ${ }^{\star}$ Indicates that the parameter is frozen.

1984), so the total model is TBABS $*$ (DISKBB + RELXILL_NK). Table 1 shows the best-fit values for model 2 and Table 2 does the same for models $2 a$ and $2 b$. The data to best-fit model ratios for models $2 a$ and $2 b$ are reported in Figure 3 and we can see that DISKBB improves the quality of the fit. However, in models $2 a$ and $2 b$ we do not recover the Kerr solution. In 


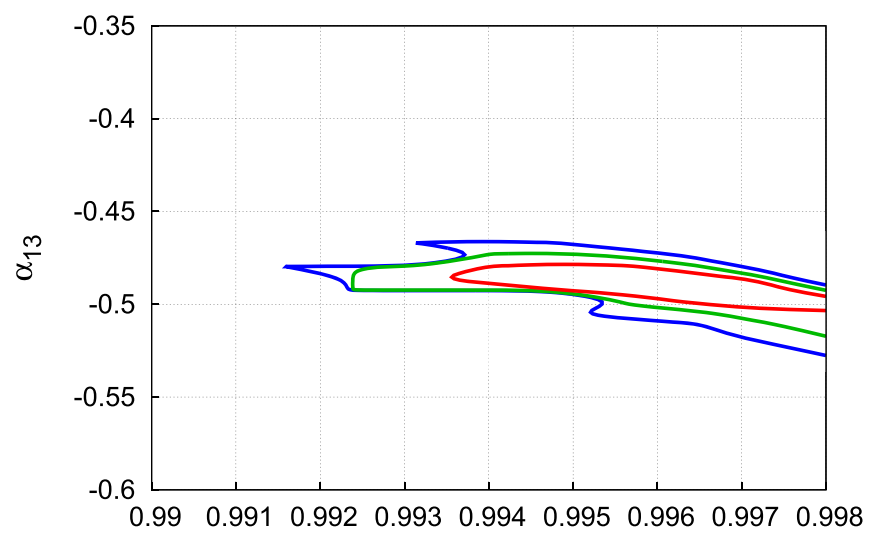

$a_{*}$

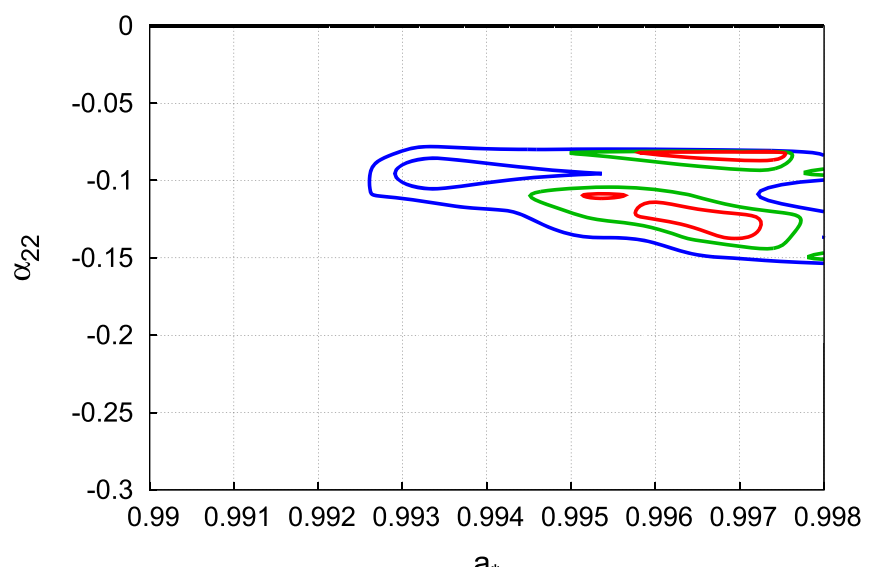

$a_{*}$

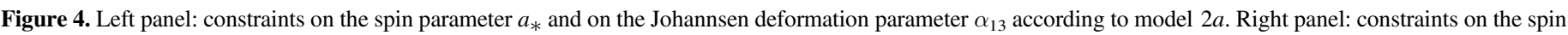

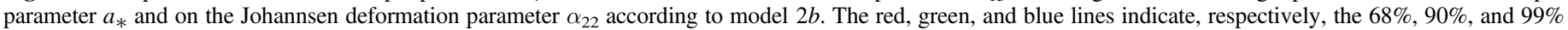
confidence level contours for two relevant parameters.

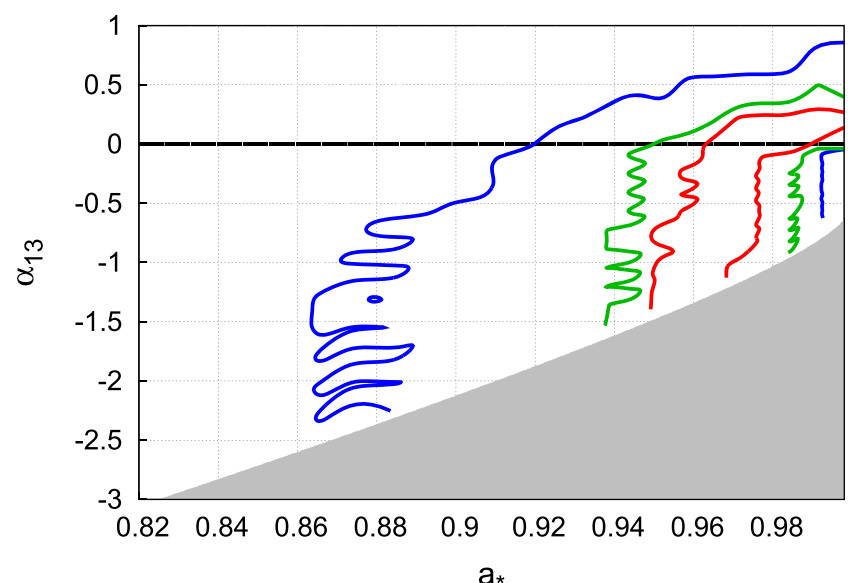

$a_{*}$

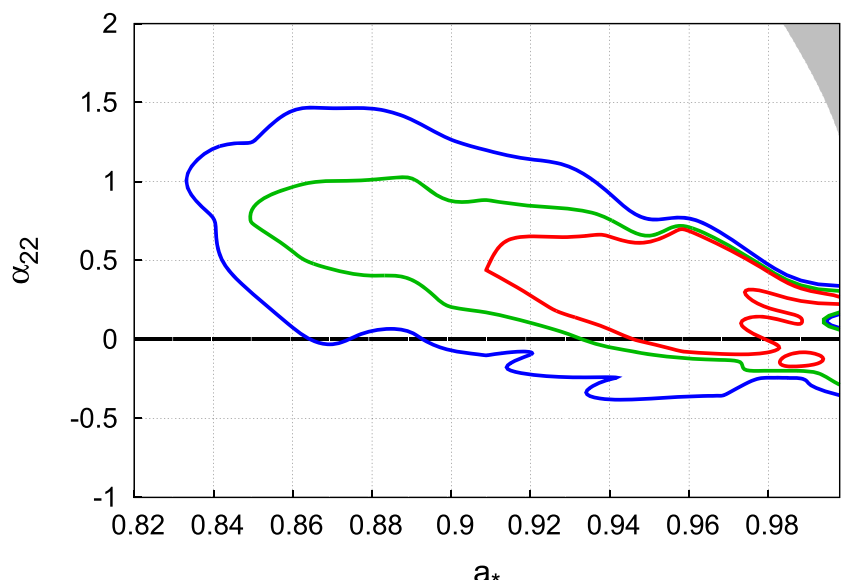

$a_{*}$

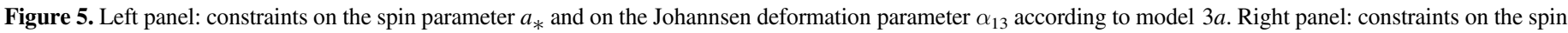

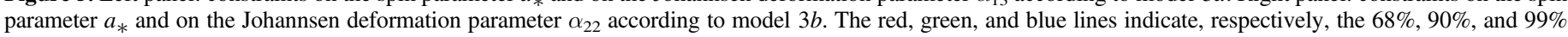

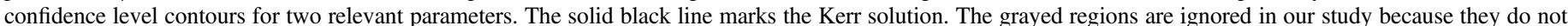
meet the conditions in Equations (4) and (5).

particular, the difference of $\chi^{2}$ between the Kerr model and model $2 a$ is $\Delta \chi^{2}=66$. The degeneracy between the spin and the deformation parameters $\alpha_{13}$ and $\alpha_{22}$ is shown in Figure 4. As discussed in $\mathrm{Xu}$ et al. (2018), the intensity profile can play an important role in the estimate of the deformation parameters. We thus try to recover the Kerr solution by fitting the data with various intensity profiles: power law, broken power law with two free emissivity indices, and broken power law with free inner emissivity index and outer emissivity index frozen to 3 . The measurement of the deformation parameters can somewhat change but, especially in the case of $\alpha_{13}$, it remains negative and far from zero by several standard deviations. It is worth noting that a pure relativistic reflection model was the model employed in Miller et al. (2013) to measure the black hole spin assuming the Kerr metric. Note also that our measurements of the model parameters for the Kerr case are consistent with those reported in Miller et al. (2013) even if there are some minor differences between the two models (Miller et al. (2013) use REFLIONX as non-relativistic reflection model while here we use XILLVER). In particular, the two spin measurements are consistent at $1 \sigma$ when we assume the Kerr metric.

We add a non-relativistic reflection component and the total model becomes TBABS $*$ (DISKBB + RELXILL_NK + XILLVER).
Such a component can be easily generated, for instance, by some outflow from the accretion disk. First, we model the emissivity profile with a simple power law: in model 3 , we assume the Kerr metric, in model $3 a$, we have $\alpha_{13}$ free and $\alpha_{22}=0$, and in model $3 b$, we have $\alpha_{13}=0$ and $\alpha_{22}$ is free. The best-fit values for the Kerr model are reported in Table 1. As shown in Table 2 and in Figure 5, the measurements of $\alpha_{13}$ and $\alpha_{22}$ are now both consistent with the Kerr solution.

Second, we employ a broken power law to describe the emissivity profile: in model $3^{\prime}$ we assume the Kerr metric, in model $3 a^{\prime}$ we have $\alpha_{13}$ free and $\alpha_{22}=0$, and in model $3 b^{\prime}$ we have $\alpha_{13}=0$ and $\alpha_{22}$ is free. As we can see from Figure 6 , the measurement of $\alpha_{22}$ is consistent with the Kerr solution. However, the measurement of $\alpha_{13}$ is very far from zero: the difference of $\chi^{2}$ between models $3^{\prime}$ and $3 a^{\prime}$ is $\Delta \chi^{2}=29$. From Table 2, we see that the inner emissivity index is lower than the outer one for models $3 a^{\prime}$ and $3 b^{\prime}$. In particular, we find $q_{\text {out }}$ very high in both cases. This simply means that the fit prefer a disk with a relatively constant emissivity near the inner edge and then a very weak emissivity at larger radii.

Table 3 lists the main models discussed above and employed in our spectral analysis, as well as the corresponding properties. 

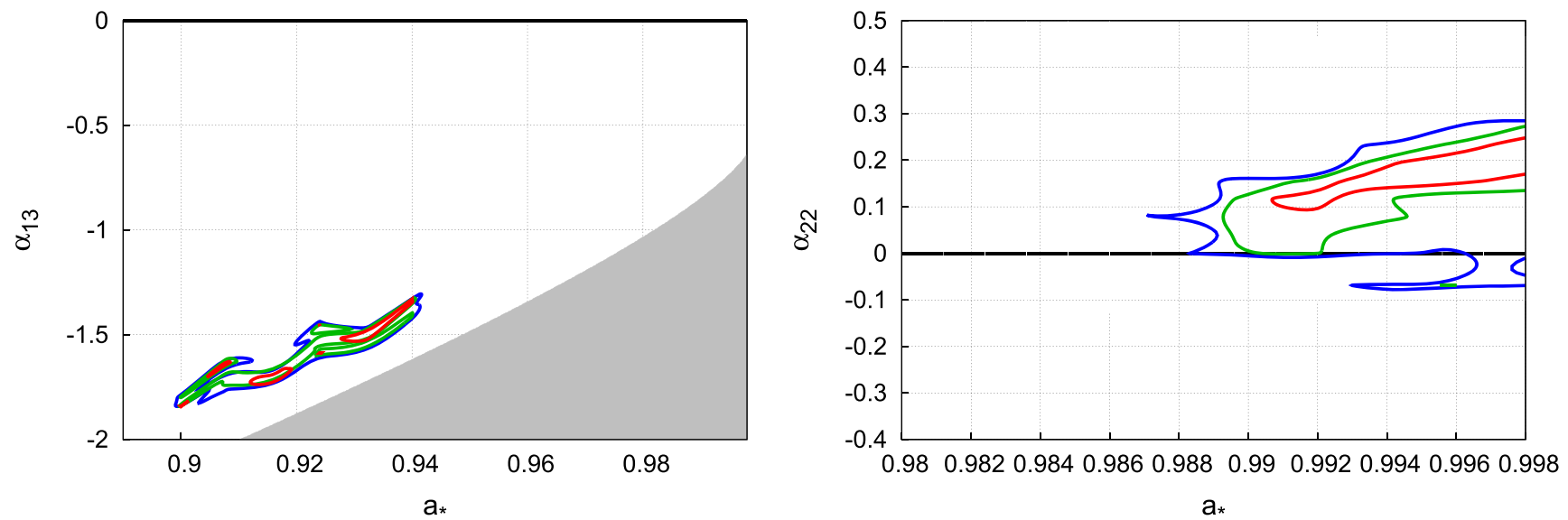

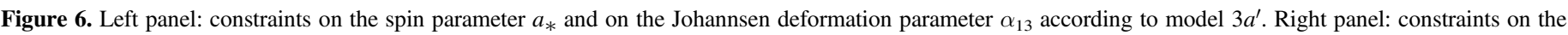

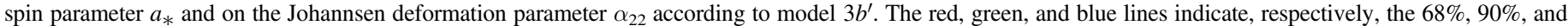

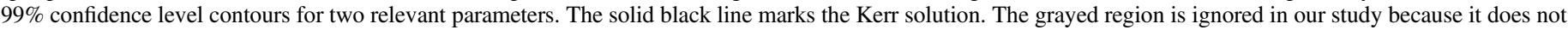
meet the condition in Equation (4).

Table 3

List of the Main Models Employed in Our Spectral Analysis

\begin{tabular}{lcc}
\hline \hline Xspec Model & Model & Description \\
\hline TBABS $*$ CUTOFFPL & 0 & \\
\hline TBABS $*$ RELXILL_NK & 1 & Kerr, $q_{\text {out }}=q_{\text {in }}$ \\
& $1 a$ & $\alpha_{13}$ free, $q_{\text {out }}=q_{\text {in }}$ \\
& $1 b$ & $\alpha_{22}$ free, $q_{\text {out }}=q_{\text {in }}$ \\
\hline TBABS $*$ (DISKBB + RELXILL_NK) & 2 & Kerr, $q_{\text {out }}=q_{\text {in }}$ \\
& $2 a$ & $\alpha_{13}$ free,,$q_{\text {out }}=q_{\text {in }}$ \\
& $2 b$ & $\alpha_{22}$ free,,$q_{\text {out }}=q_{\text {in }}$ \\
\hline TBABS $*$ (DISKBB + RELXILL_NK + XILLVER) & 3 & Kerr, $q_{\text {out }}=q_{\text {in }}$ \\
& $3 a$ & $\alpha_{13}$ free,,$q_{\text {out }}=q_{\text {in }}$ \\
& $3 b$ & $\alpha_{22}$ free,,$q_{\text {out }}=q_{\text {in }}$ \\
\hline TBABS $*(D I S K B B+$ RELXILL_NK + XILLVER) & $3^{\prime}$ & Kerr, $q_{\text {out }}$ free \\
& $3 a^{\prime}$ & $\alpha_{13}$ free,,$q_{\text {out }}$ free \\
& $3 b^{\prime}$ & $\alpha_{22}$ free,,$q_{\text {out }}$ free \\
\hline
\end{tabular}

\section{Discussion and Conclusions}

When we add a non-relativistic reflection component to the model, the quality of the fit improves, and we can argue that such a non-relativistic reflection component is indeed necessary. It is thus perfectly understandable that we do not recover the Kerr solution in models $2 a, 2 b$, and their variants with a different emissivity profile. We are missing an important component in the spectrum and we cannot pretend to test the Kerr metric with GRS $1915+105$. The take-away message is that the choice of the correct model can be very important in these kinds of tests.

With the non-relativistic reflection component in the model, the measurements of $\alpha_{13}$ and $\alpha_{22}$ turn out to be very sensitive to the choice of the shape of the emissivity profile. In models $3 a$ and $3 b$, we recover the Kerr solution, but the constraints on $\alpha_{13}$ and $\alpha_{22}$ are weak. In model $3 a^{\prime}$, we do not recover the Kerr solution at a high confidence level. In model $3 b^{\prime}$, we recover the Kerr solution and the constraint is strong. Note that we cannot say that the correct astrophysical model is the one in which we recover the Kerr metric, because this would be equivalent to saying that we want to test the astrophysical model and we assume the Kerr metric. We have thus to figure out how we can separately test the metric and the astrophysical model.

We note that we are not able to fit well the high-energy part of the spectrum (see Figure 3). Since the cut-off energies that we obtain are very low, the corona should be relatively cool. In such a case, the difference between a simple cut-off power law and a proper comptonization model may be important. We have thus repeated our analyses by replacing RELXILL_NK with RELXILLCP_NK in our models (Abdikamalov et al. 2019). However, we have obtained worse fits. We have also tried other solutions, like adding an extra power-law component to describe the possible emission from the jet, but still we are not able to improve the quality of the fit at high energies.

In our previous analyses of stellar-mass and supermassive black holes with RELXILL_NK, we had never found similar problems. First, we were able to easily recover vanishing values of $\alpha_{13}$ and $\alpha_{22}$. Second, the choice between power law and broken power law for the description of the intensity profile had not such a strong impact on the final estimate of $\alpha_{13}$ and $\alpha_{22}$. More specifically, we usually found that a power law or a broken power law could provide somewhat different but consistent results. On the contrary, imposing an ad hoc emissivity profile (i.e., without fitting the emissivity indices and the breaking radius), we obtained non-vanishing values of $\alpha_{13}$ and $\alpha_{22}$. With such results, we argued that the emissivity profile is important to correctly model the spectrum of the source, but that it is possible to separately measure the deformation parameters and the parameters related to the emissivity profile. The case of GRS $1915+105$ seems to be different.

It is likely that the spectrum of GRS $1915+105$ is more difficult to model. While the spectrum may indeed be described by a thermal component from the disk and relativistic and nonrelativistic reflection components, RELXILL_NK and XILLVER may not be able to properly describe these components. Both models have indeed a number of simplifications. If the theoretical model does not properly describe the observed spectrum, the fit tries to absorb such a discrepancy with incorrect values of the parameters.

A crucial assumption in our reflection model RELXILL_NK is that the accretion disk is thin and the inner edge is at the ISCO radius. If the actual accretion disk around the black hole does not meet these conditions, we can have systematic uncertainties 
that can mimic a non-vanishing deformation parameter. However, we do not think this is the reason for our results for GRS $1915+105$. For the NuSTAR observation of 2012, the Eddington-scaled accretion luminosity of the source is $0.23 \pm 0.04$ (Miller et al. 2013), which is in the 0.05-0.30 range required to have thin disks (Steiner et al. 2010; McClintock et al. 2014). On the contrary, in other works we have obtained quite stringent constraints on $\alpha_{13}$ and $\alpha_{22}$ from supermassive black holes that more likely accrete above $30 \%$ of their Eddington limit.

The current version of XILLVER is appropriate for the description of "cold" disks, because it neglects the contribution in the reflection spectrum from the X-ray photons emitted by the disk itself. This may explain our difficulties in recovering the Kerr metric. In the case of GS 1354-645, the fit did not need any thermal component, which means that the disk's temperature is lower than the one in the NUSTAR observation of GRS $1915+105$. In the case of supermassive black holes, the temperature of the disk is a few orders of magnitude lower, so XILLVER is appropriate. We note that we are encountering a similar problem in recovering the Kerr solution in a work in preparation on Cygnus X-1, where we analyze some NuSTAR observations in which the source is in the soft state.

Lastly, we note that in all our models, we have always assumed that the inner edge of the accretion disk is at the ISCO radius, i.e., $R_{\text {in }}=R_{\mathrm{ISCO}}$. This is quite a common choice, both when we try to measure the black hole spin assuming the Kerr metric and when we want to test the Kerr hypothesis. If we do not do so, in general, it is impossible to measure the spin and the deformation parameter because of the strong degeneracy of the latter with $R_{\text {in }}$. However, this is not the case when the black hole is rotating very fast, with a spin parameter close to the maximum value allowed by the model, such as for GRS 1915 +105 . As we have already shown in $\mathrm{Xu}$ et al. (2018) for GS 1354-645 and the Johannsen metric, leaving $R_{\text {in }}$ as a free parameter has quite a negligible impact on the estimate of the other model parameters, because $R_{\text {in }}$ is already constrained to be close to the minimum value allowed by the model. Note that such a conclusion cannot be automatically extended to any metric.

The possibility of performing precise tests of the Kerr metric using X-ray reflection spectroscopy, which is our long-term goal, requires having a theoretical model that is good enough such that the systematic uncertainties are subdominant with respect to the statistical ones. In such a case, it is important to be able to select the correct candidates, namely, observations of black holes that can be well described by the available theoretical model. This will become of crucial importance with the next generation of X-ray missions, like eXTP and Athena, that promise to provide unprecedented high-quality data (Zhang et al. 2016).

This work was supported by the National Natural Science Foundation of China (NSFC), grant No. U1531117, and Fudan University, grant No. IDH1512060. Y.Z. also acknowledges the support from the Fudan Undergraduate Research
Opportunities Program (FDUROP). A.B.A. also acknowledges the support from the Shanghai Government Scholarship (SGS). J.A.G. acknowledges support from the Alexander von Humboldt Foundation. S.N. acknowledges support from the Excellence Initiative at Eberhard-Karls Universität Tübingen.

\section{ORCID iDs}

Cosimo Bambi (iD https://orcid.org/0000-0002-3180-9502

\section{References}

Abbott, B. P., Abbott, R., Abbott, T. D., et al. 2016, PhRvL, 116, 221101 Abdikamalov, A. B., Ayzenberg, D., Bambi, C., et al. 2019, arXiv:1902.09665 Arnaud, K. A. 1996, adass V, 101, 17

Bambi, C. 2013a, PhRvD, 87, 023007

Bambi, C. 2013b, PhRvD, 87, 084039

Bambi, C. 2017, RvMP, 89, 025001

Bambi, C. 2018, AnP, 530, 1700430

Bambi, C., Abdikamalov, A., Ayzenberg, D., et al. 2018, Univ, 4, 79

Bambi, C., Cárdenas-Avendaño, A., Dauser, T., García, J. A., \& Nampalliwar, S. 2017, ApJ, 842, 76

Bambi, C., Jiang, J., \& Steiner, J. F. 2016, CQGra, 33, 064001

Cao, Z., Nampalliwar, S., Bambi, C., Dauser, T., \& García, J. A. 2018, PhRvL, 120,051101

Cardoso, V., \& Gualtieri, L. 2016, CQGra, 33, 174001

Choudhury, K., Nampalliwar, S., Abdikamalov, A. B., et al. 2018, arXiv:1809. 06669

Dauser, T., Garcia, J., Wilms, J., et al. 2013, MNRAS, 430, 1694

Dauser, T., Wilms, J., Reynolds, C. S., \& Brenneman, L. W. 2010, MNRAS, 409, 1534

Fabian, A. C., Iwasawa, K., Reynolds, C. S., \& Young, A. J. 2000, PASP, 112,1145

García, J., Dauser, T., Lohfink, A., et al. 2014, ApJ, 782, 76

García, J., Dauser, T., Reynolds, C. S., et al. 2013, ApJ, 768, 146

García, J., \& Kallman, T. R. 2010, ApJ, 718, 695

Jiang, J., Bambi, C., \& Steiner, J. F. 2015, ApJ, 811, 130

Johannsen, T. 2013, PhRvD, 88, 044002

Johannsen, T., \& Psaltis, D. 2013, ApJ, 773, 57

Krawczynski, H. 2018, GReGr, 50, 100

McClintock, J. E., Narayan, R., \& Steiner, J. F. 2014, SSRv, 183, 295

Miller, J. M., Parker, M. L., Fuerst, F., et al. 2013, ApJL, 775, L45

Mitsuda, K., Inoue, H., Koyama, K., et al. 1984, PASJ, 36, 741

Narayan, R. 2005, NJPh, 7, 199

Ni, Y., Zhou, M., Cárdenas-Avendaño, A., et al. 2016, JCAP, 7, 049

Reynolds, C. S. 2014, SSRv, 183, 277

Schee, J., \& Stuchlík, Z. 2009, GReGr, 41, 1795

Steeghs, D., McClintock, J. E., Parsons, S. G., et al. 2013, ApJ, 768, 185

Steiner, J. F., McClintock, J. E., Remillard, R. A., et al. 2010, ApJL, 718, L117

Tripathi, A., Nampalliwar, S., Abdikamalov, A. B., et al. 2018a, PhRvD, 98, 023018

Tripathi, A., Nampalliwar, S., Abdikamalov, A. B., et al. 2018b, arXiv:1811. 08148

Tripathi, A., Yan, J., Yang, Y., et al. 2019, ApJ, 874, 135

Wang-Ji, J., Abdikamalov, A. B., Ayzenberg, D., et al. 2018, arXiv:1806. 00126

Will, C. M. 2014, LRR, 17, 4

Wilms, J., Allen, A., \& McCray, R. 2000, ApJ, 542, 914

Xu, Y., Nampalliwar, S., Abdikamalov, A. B., et al. 2018, ApJ, 865, 134

Yagi, K., \& Stein, L. C. 2016, CQGra, 33, 054001

Yunes, N., \& Siemens, X. 2013, LRR, 16, 9

Yunes, N., Yagi, K., \& Pretorius, F. 2016, PhRvD, 94, 084002

Zhang, S. N., Feroci, M., Santangelo, A., et al. 2016, Proc. SPIE, 9905, 99051Q

Zhou, M., Cardenas-Avendano, A., Bambi, C., Kleihaus, B., \& Kunz, J. 2016, PhRvD, 94, 024036 